\title{
SOLAR AND SUPERNOVA CONSTRAINTS ON COSMOLOGICALLY INTERESTING NEUTRINOS
}

\author{
W. C. HAXTON \\ Institute for Nuclear Theory, Box 351550 \\ and Department of Physics, Box 351560 \\ University of Washington, Seattle, Washington 98195 USA
}

\begin{abstract}
The sun and core-collapse supernovae produce neutrino spectra that are sensitive to the effects of masses and mixing. Current results from solar neutrino experiments provide perhaps our best evidence for such new neutrino physics, beyond the standard electroweak model. I discuss this evidence as well as the limited possibilities for more conventional explanations. If the resolution of the solar neutrino problem is $\nu_{e} \rightarrow \nu_{\mu}$ oscillations, standard seesaw estimates of $m_{\nu_{\tau}}$ suggest a cosmologically interesting third-generation neutrino. I discuss recent nucleosynthesis arguments that lead to an important constraint on this possibility.
\end{abstract}




\section{INTRODUCTION}

The two special lectures presented at Erice under somewhat different titles are combined here under the unifying theme I stressed at the school: What can we learn about neutrino masses and mixing from solar and Type II supernova studies, and what constraints follow for massive neutrinos in cosmology and large-scale structure? The first half of this paper contains a brief review of the current status of the solar neutrino problem and of the evidence for matter-enhanced neutrino mixing. I argue that the alternative of a nonstandard solar model is now limited to a single but very interesting class of solutions where the core mixes on timescales comparable to those for pp chain ${ }^{3} \mathrm{He}$ equilibration. Oscillation of the $\nu_{e}$ with the $\nu_{\mu}$ is a particularly intriguing solution to the solar neutrino problem, as seesaw estimates of the $m_{\nu_{\tau}}$ then place it near or within the range of cosmologically interesting values. However, this possibility may be quite constrained due to growing evidence that r-process nucleosynthesis occurs deep within a Type II supernova. Massive tauon neutrinos with even modest mixing angles can destroy the conditions necessary for the r-process. This leads to a somewhat distressing situation where massive tauon neutrinos, if helpful cosmologically, may have properties that render direct detection particularly difficult. Thus the prospect of continuing hot dark matter uncertainties in large scale structure simulations may be quite real.

One of the experimental pillars of modern cosmology is the nucleosynthesis of the light elements within the first few minutes of the big bang. A second reason, in addition to $m_{\nu_{\tau}}$, for sharing the r-process story with the students at this school is to stress its similarities to big bang nucleosynthesis. Each process involves expanding, radiation-dominated nucleon gases, nuclear freezeout, and the complications of having to deduce initial conditions from a "fossil" record of abundances. But the dividends that can follow from a detailed understanding of the underlying mircrophpysics is, in each case, most significant: from He synthesis we learn about the baryon/photon ratio $\eta$ and the number of light neutrino generations; from the r-process we may learn about the mass and mixing angles of the $\nu_{\tau}$.

\section{SOLAR NEUTRINOS: WHERE ARE WE?}

The original motivation for measuring solar neutrinos was the opportunity to make a quantitative check on our theories of stellar evolution and nuclear energy generation. The neutrino fluxes produced by our sun can be measured and compared to the predictions of the standard solar model (SSM) [1,2]. This model traces solar evolution from the onset of the main sequence, assuming hydrostatic equalibrium (local balance between the gravitational force and the gas pressure gradient); energy transport by radiation (interior) and convection (outer envelope); and solar energy generation by fusion. The input microphysics includes the opacity as a function of temperature and composition, and the nuclear reaction rates for the dominant pp chain and CNO cycle conversion of four protons into ${ }^{4} \mathrm{He}$. As nuclear reaction energies in the sun are typically $\sim 10 \mathrm{keV}$, the nuclear S-factors have to be deduced from somewhat higher energy terrestrial cross section measurements by extrapolation. This requires theory to provide the shape $\mathrm{S}(\mathrm{E})$ of these nonresonant reactions and to correct for the effects of atomic screening in terrestrial targets. Finally, the model must satisfy the boundary conditions of the present day sun (mass, luminosity, radius); the solar age 
( $\sim 4.6 \mathrm{~Gy}$ ); and initial metallicity (abundances for $\mathrm{A}>4$ are equated to today's surface abundances, under the assumption that the surface has been undisturbed).

The resulting sun is a dynamic one with a long-term luminosity rise of $\sim 44 \%$, a ${ }^{8} \mathrm{~B}$ neutrino flux that has been significant only in the last $10^{9}$ years $\left(\phi\left({ }^{8} \mathrm{~B}\right) \sim \phi_{o} e^{\tau / \tau_{o}}, \tau_{o} \sim 0.9 \mathrm{~Gy}\right)$, and interesting nuclear burning scales, such as the time for reaching ${ }^{3} \mathrm{He}$ equilibration in the pp chain. Notable successes of the standard solar model include the correspondence between the predicted ${ }^{4} \mathrm{He}$ abundance near the solar surface and the value derived from helioseismology, and the correct depth of the convective zone (compare to the value deduced from p-mode oscillations) [1]. Among the SSM simplifying assumptions are its one-dimensional form, which allows no mixing even during the sun's early convective phase. One of the often noted shortcomings of the SSM, its failure to explain the observed Li depletion by a factor $\sim 100$, is presumably associated with this assumption.

SSM helium synthesis occurs $\sim 98 \%$ of the time through the pp chain, illustrated in Fig. 1. The chain is comprised of three cycles (ppI, ppII, ppIII) corresponding to three distinct terminations of the fusion. The ppII and ppIII cycles are "tagged" by associated neutrinos, those from ${ }^{7} \mathrm{Be}$ electron capture and ${ }^{8} \mathrm{~B} \beta$-decay, respectively, while the overall rate of hydrogen burning is given by the rate of pp and pep neutrinos. The competition between these three cycles is a detailed test of conditions - temperature and composition - within the solar core. Thus the detection of the associated neutrinos - measuring the fluxes and spectra illustrated in Fig. 2 - provides an important check on the SSM.

The heroic effort to measure these fluxes began with the ${ }^{37} \mathrm{Cl}$ detector [3] and continued with Kamiokande II/III [4] and Superkamiokande [5] and with the SAGE/GALLEX gallium experiments [6,7]. It continues with the mounting of the Sudbury Neutrino Observatory [8], which will begin operations early in 1998. A fit of the fluxes to existing results yields a surprising pattern

$$
\begin{aligned}
\phi(\mathrm{pp}) & \sim \phi_{\mathrm{SSM}}(\mathrm{pp}) \\
\phi\left({ }^{7} \mathrm{Be}\right) & \sim 0 \\
\phi\left({ }^{8} \mathrm{~B}\right) & \sim 0.4 \phi_{\mathrm{SSM}}\left({ }^{8} \mathrm{~B}\right),
\end{aligned}
$$

where the subscripts SSM denote SSM values. These departures from expected values lie far outside the known ranges of SSM uncertainties. (See Fig. 4 of Ref.[9]).

There have been many attempts to modify the SSM in order to improve the agreement with the results in Eq. (1). Such "nonstandard" SMs include ad hoc changes in SSM parameters far outside their accepted uncertainties (e.g., increasing $\mathrm{S}_{11}$, the S-factor for the $\mathrm{p}+\mathrm{p}$ reaction) as well as new physics assumptions (e.g., mechanisms resulting in a significantly reduced heavy element abundance in the solar core). The results of a series of such calculations are shown in Fig. 3, taken from Hata et al. [10]. Systematically one finds that $\phi\left({ }^{7} \mathrm{Be}\right)$ can be suppressed only at the cost of an even larger suppression of $\phi\left({ }^{8} \mathrm{~B}\right)$, in contrast to Eq. (1). That is, the trajectory of $\phi\left({ }^{8} \mathrm{~B}\right)-\phi\left({ }^{7} \mathrm{Be}\right)$ fluxes in Fig. 3 follows a path below diagonal, while the experimental results are above the diagonal (Another nice illustration of this is found in Castellani et al. [11].)

This difficulty is due to the dependence of the ppII and ppIII cycles on the solar core temperature $T_{c}$. As $\phi\left({ }^{8} \mathrm{~B}\right) \sim T_{c}^{21}$, the observed depletion of $\phi\left({ }^{8} \mathrm{~B}\right)$ in the water Cerenkov experiments requires 


$$
T_{c} \sim 0.96 T_{c}^{\mathrm{SSM}},
$$

that is, a cooler core. But $\phi\left({ }^{7} \mathrm{Be}\right) / \phi\left({ }^{8} \mathrm{~B}\right) \sim T_{c}^{-10}$, so that such a reduced temperature implies

$$
\frac{\phi\left({ }^{7} \mathrm{Be}\right)}{\phi\left({ }^{8} \mathrm{~B}\right)} \sim 1.5 \frac{\phi^{\mathrm{SSM}}\left({ }^{7} \mathrm{Be}\right)}{\phi^{\mathrm{SSM}\left({ }^{8} \mathrm{~B}\right)}},
$$

in contradiction to Eq. (1). In other words, the reduced $\phi\left({ }^{8} \mathrm{~B}\right)$ and reduced flux ratio $\phi\left({ }^{7} \mathrm{Be}\right) / \phi\left({ }^{8} \mathrm{~B}\right)$ apparent from Eq. (1) are in conflict, with the first requiring a cooler core and the second a hotter one.

\section{MATTER-ENHANCED NEUTRINO OSCILLATIONS}

If this argument is completely robust, it appears that current experimental results cannot be accommodated by changing the SSM, but instead require new particle physics. While many suggestions have been made, the solution almost universely favored, due to its simplicity and plausibility, is neutrino oscillations enhanced by matter effects (the MikheyevSmirnov-Wolfenstein mechanism) [12].

Specializing to the two-flavor case, neutrino oscillations occur if the weak interaction eigenstates

$$
\left|\nu_{e}\right\rangle,\left|\nu_{\mu}\right\rangle
$$

defined in terms of their accompanying charged leptons, do not correspond to the mass eigenstates which diagonalize the free Hamiltonian

$$
\left|\nu_{L}\right\rangle,\left|\nu_{H}\right\rangle
$$

with mass $m_{L}$ (light) and $m_{H}$ (heavy). Instead there is a nontrivial rotation between these two bases, so that

$$
\left|\nu_{p}(0)\right\rangle=\left|\nu_{e}\right\rangle=\cos \theta_{v}\left|\nu_{L}\right\rangle+\sin \theta_{v}\left|\nu_{H}\right\rangle,
$$

where $\left|\nu_{p}(0)\right\rangle$ is the neutrino of momentum $p$ produced at time $\mathrm{t}=0$ by $\beta$-decay. The particle physics prejudice that $\left|\nu_{e}\right\rangle$ should be primarily composed of the light mass eigenstate suggests $\theta_{v}$ is small. A simple calculation yields downstream of the $\beta$ decay source a probability of observing a $\nu_{\mu}$,

$$
\left|\left\langle\nu_{\mu} \mid \nu_{p}(t)\right\rangle\right|^{2}=\sin ^{2} 2 \theta_{v} \sin ^{2}\left(\frac{\delta m^{2}}{4 E} t\right),
$$

where $\delta m^{2}=m_{H}^{2}-m_{L}^{2}$. Thus such vacuum oscillations yield a small $\left|\nu_{\mu}\right\rangle$ appearance probability proportional to $\sin ^{2} 2 \theta_{v}$.

Matter, however, can act as a marvelous regenerator, enhancing this oscillation probability because of an adiabatic level crossing. The neutrino index of refraction is modified in matter by charged and neutral current interactions, and this effect is flavor dependent because the charge current interactions with solar electrons only contributes to the $\nu_{e}$ forward 
scattering amplitude. The result is a contribution to the mass matrix $M^{2}$ in the flavor basis of

$$
\left(M^{2}\right)_{\nu_{e} \nu_{e}}=4 E \sqrt{2} G_{F} \rho_{e}(x)
$$

where $E$ is the neutrino energy and $\rho_{e}(x)$ the local density of electrons. That is, the electron neutrino becomes heavier at high density.

The resulting MSW phenomenon is illustrated in Fig. 4, where $m_{H}^{2}(x) / 2 \mathrm{E}$ and $m_{L}^{2}(x) / 2 E$, which are now functions of $x$ because of their dependence on $\rho_{e}(x)$, are plotted relative to their average value. The relationship between the local mass eigenstates $\left|\nu_{L}(x)\right\rangle$ and $\left|\nu_{H}(x)\right\rangle$, corresponding to $m_{L}(x)$ and $m_{H}(x)$, and $\left|\nu_{e}\right\rangle$ is given by Eq. (6), with the important change that $\theta(x)$ now depends on $x$. At $\rho=0, \theta(x)=\theta_{v} \sim 0$, as we have assumed, because of our particle physics prejudices, that $\left|\nu_{e}\right\rangle \sim\left|\nu_{L}\right\rangle$ in vacuum. But as Eq. (8) is a positive contribution to $\left(M^{2}\right)_{\nu_{e} \nu_{e}}$, at sufficiently high density $\left|\nu_{H}(x)\right\rangle \rightarrow\left|\nu_{e}\right\rangle$. That is, as the density increases, $\theta(x)$ rotates from $\theta_{\nu} \sim 0$ to $\sim \pi / 2$. Furthermore, there is an intermediate critical density $\rho\left(x_{c}\right)$ where the matter effects just cancel the vacuum mass difference between $\left(M^{2}\right)_{\nu_{e} \nu_{e}}$ and $\left(M^{2}\right)_{\nu_{\mu} \nu_{\mu}}$, leading to the avoided level crossing of Fig. 4.

The transformation to local mass eigenstates leads to a wave equation that is diagonal apart from terms depending on $\mathrm{d} \rho(x) / d x$. But if a $\left|\nu_{e}\right\rangle$ is produced at $\rho\left(x_{i}\right)>\rho\left(x_{c}\right)$ and $d p(x) / d x$ is everywhere ignorable (i.e., $\operatorname{d} \ln (\rho) / d x$ is small over lengths comparable to the inverse splittings of the local mass eigenstates of Fig. 4, the propagation is adiabatic. This corresponds to remaining on the heavy mass trajectory in Fig. 4, transforming the $\left|\nu_{e}\right\rangle$ into a $\left|\nu_{\mu}\right\rangle$. Thus nearly complete $\left|\nu_{e}\right\rangle \rightarrow\left|\nu_{\mu}\right\rangle$ conversion will occur if [9]: $\delta m^{2}$.

1) The initial density $\rho\left(x_{i}\right)$ is sufficient to generate the level crossing, $4 E \sqrt{2} G_{F} \rho_{e}(x) \gg$

2) The propagation is adiabatic. As the separation between mass eigenstates is a minimum at $x_{c}$, this is the point where the oscillation wavelength is maximum. Thus changes in $\rho(x)$ can best be "seen" at the point. One anticipates, therefore, that the adiabatic condition is most severe at the avoided level crossing.

All of this can be worked out analytically using the Landau-Zener trick, as described in Ref. [9]. The resulting $\nu_{e}$ survival probability is

$$
P_{\nu_{e}}^{L Z}=\frac{1}{2}+\frac{1}{2} \cos 2 \theta_{v} \cos 2 \theta_{i}\left(1-2 P_{\text {hopping }}\right),
$$

where $P_{\text {hopping }}$ is the probability for jumping to the light mass trajectory,

$$
P_{\text {hopping }}=e^{-\pi \gamma_{c} / 2} \text {, }
$$

and

$$
\gamma_{c}=\frac{\sin ^{2} 2 \theta_{v}}{\cos 2 \theta_{v}} \frac{\delta m^{2}}{2 E} \frac{1}{\left|\frac{1}{\rho_{c}} \frac{d \rho(x)}{d x}\right|_{x=x_{c}} \mid} .
$$

Note that $\gamma_{c}$ depends on the density derivative at the crossing point. The adiabatic limit $\left(P_{\text {hopping }}=0\right.$ in Eq. (9a)) was derived by Bethe [13], while $P_{\text {hopping }}$ was derived by Haxton [14] and independently by Parke [15]. The two conditions above correspond to the initial local mixing angle $\theta_{i} \sim \pi / 2$ and to $\gamma_{c} \gg 1$, yielding $P_{\nu_{e}}^{L Z} \sim \frac{1}{2}-\frac{1}{2} \cos 2 \theta_{v} \sim 0$. 
A search for a fit to the experimental results gives the familiar iso-SNU plot shown in Fig. 5. The better fit to the data is given by the small mixing angle solution of $\sin ^{2} 2 \theta_{v} \sim$ 0.005 and $\delta m^{2} \sim 6 \cdot 10^{-6} \mathrm{eV}^{2}$. This corresponds to strong conversion of the intermediate energy ${ }^{7} \mathrm{Be}$ neutrinos; partial depletion of the ${ }^{8} \mathrm{~B}$ neutrinos, strongest at the low energy end; and survival of most of the pp neutrinos. This occurs because the ${ }^{7}$ Be neutrinos have an adiabatic level crossing; almost all of the lower energy pp neutrinos do not have a level crossing and thus evade conversion; while the higher energy ${ }^{8} \mathrm{~B}$ neutrinos straddle the adiabatic boundary, so that the lower energy portion of the spectrum is converted more strongly than the higher energy end. Thus the MSW mechanism has a dramatic signature, a characteristic energy-dependent distortion of the $\nu_{e}$ spectrum and the appearance of $\nu_{\mu}$ neutrinos.

If this is the solution to the solar neutrino problem, it may have important consequences for those working in cosmology and large scale structure. Generalizing the above discussion to three mass eigenstates, with $m_{1} \ll m_{2} \ll m_{3}$ and $\left|\nu_{1}\right\rangle \sim\left|\nu_{e}\right\rangle$, then an attractive choice is $m_{2} \sim \sqrt{m_{2}^{2}-m_{1}^{2}} \sim \sqrt{\delta m^{2}} \sim$ few $\cdot 10^{-3} \mathrm{eV}$. That is, if we suppose the oscillation is $\nu_{e} \rightarrow \nu_{\mu}$ rather than $\nu_{e} \rightarrow \nu_{\tau}$, we can estimate the "muon" neutrino mass.

If one thinks in terms of the more general multiplets that might exist in extensions of the standard model, e.g.,

$$
\left(\begin{array}{c}
u \\
d \\
\nu_{e} \\
e^{-}
\end{array}\right)
$$

the much smaller mass of the $\nu_{e}$ relative to the other first-generation fermions is a bit of a puzzle. One would have assumed that all of these particles will have similar couplings to the mass-generating fields, and thus might have comparable masses $\sim m_{D}$. One nice resolution of this problem comes from the observation that $\nu_{e}$ is unique in not having a charge - or any other additively conserved quantum number.

Thus neutrinos can also have Majorana masses, e.g.,

$$
M_{R} \overline{\nu_{R}^{c}} \nu_{R}
$$

If the right-handed Majorana mass is large, characterizing the scale of some new physics, the resulting diagonalization of the mass matrix yields one heavy eigenstate and one light one

$$
\sim m_{D}\left(\frac{m_{D}}{M_{R}}\right)
$$

This "seesaw" mechanism [16] thus generates the needed small parameter, $m_{D} / m_{R}$, to explain the lightness of the neutrino relative to its charged partners. Now Eq. (12) suggests that the mass relations $m_{\nu_{e}}: m_{\nu_{\mu}}: m_{\nu_{\tau}}$ might be related to the squares of the Dirac masses for the corresponding upper isospin quarks, $m_{u}^{2}: m_{c}^{2}: m_{\tau}^{2}$. Thus " $m_{\nu_{\mu}}$ " $\sim$ few $\cdot 10^{-3} \mathrm{eV}$ allows us to estimate the $M_{R}$ and predict $m_{\nu_{\tau}} \sim(1-$ few $) \mathrm{eV}$. That is, an MSW $\nu_{e} \rightarrow \nu_{\mu}$ solution to the solar neutrino problem is attractive because it allows the $\nu_{\tau}$ to play a cosmologically interesting role, perhaps being a source of hot dark matter helpful in explaining the formation of large-scale structure. 


\section{IS THERE NO ALTERNATIVE TO NEW PARTICLE PHYSICS?}

Because the conclusion reached in the last section is a profound one, having far-reaching consequences for both particle physics and cosmology, it is important to ask whether there is any more conventional "escape". Andrew Cumming and I recently found that there is one exception to the $T_{c}$ arguments in Eqs. (2) and (3), and thus one possible path to a nonstandard model that might accommodate Eq. (1). Because this work is described in a publication and in another talk [17], I will present only a brief summary here.

As illustrated in Fig. 3, many SSM modifications have been tried and found to depart from the pattern in Eq. (1). This suggested to us that if an acceptable nonstandard SSM possibility exists, its underlying physics might be subtle and thus difficult to anticipate. Therefore we decided to try a naive approach, exploring nonstandard SMs phenomenologically, in the hope that this might point the way to the necessary change in the SSM.

As we wanted our explorations to be reasonable, we required that our phenomenological adjustments preserve certain properties. This included reproducing the correct luminosity; retaining SSM microphysics, given the work that has been invested in determining accurate S-factors and opacities; and demanding that the model be steady-state. The motivation for the last condition was to avoid solutions where today is somehow a special time in the sun's history.

In the SSM the steady-state condition is satisfied locally: the production of various "catalysts" of the pp chain like $\mathrm{d},{ }^{3} \mathrm{He}$, and ${ }^{7} \mathrm{Be}$ is equated to the local consumption, once equilibrium is reached. This is not a physics result in the SSM, but a consequence of its assumptions (no mixing in one dimension). Thus the "models" we explored phenomenologically were considerably less constrained, allowing transport of the "catalysts" mentioned above as well as $\mathrm{H}$ and ${ }^{4} \mathrm{He}$.

Our procedures are described in [17] and will not be repeated here. They led us to focus quickly on ${ }^{3} \mathrm{He}$, which offers the prospect of transport on interesting time scales. Equating the production rate of ${ }^{3} \mathrm{He}$ through the $\mathrm{p}+\mathrm{p}$ reaction to its primary destruction rate, ${ }^{3} \mathrm{He}$ $+{ }^{3} \mathrm{He}$, leads to an estimate of the SSM equilibrium abundance

$$
X_{3}^{e q} \sim 7 \cdot 10^{-4} X_{1} T_{7}^{-6}
$$

and of the time required to reach equilibrium

$$
\tau_{3}^{e q} \sim \frac{1}{X_{1}} T_{7}^{-10}
$$

where $X_{1}$ is the local abundance of $\mathrm{H}$ and $T_{7}$ the temperature in $10^{7} \mathrm{~K}$. For example, $99 \%$ of equilibrium is reached at $\mathrm{r} \sim 0.1 R_{\odot}$ after $\sim 5 \cdot 10^{6}$ years of SSM burning, and at $\mathrm{t}=$ 4.6 Gy equilibrium has been achieved for $\mathrm{r} \lesssim 0.27 R_{\odot}$. The resulting ${ }^{3} \mathrm{He} \mathrm{SSM}$ profile is thus characterized by a steep gradient over the energy-producing core, shown in Fig. 6 .

Our exercise showed that a steady-state solar model can produce neutrino fluxes reasonably close to Eq. (1) only if the core mixes in a prescribed way on a timescale characteristic of ${ }^{3} \mathrm{He}$ equilibration, as illustrated in Fig. 7. The circulation is that of "elevator convection": localized, relative rapid downward flow of ${ }^{3} \mathrm{He}$ rich material in narrow plumes, followed by a slower, broad restoring flow to large $\mathrm{r}$. 
It is important to understand on general grounds, why such circulation would produce a flux pattern similar to Eq. (1). First, the competition between the ppI cycle and the ppII + ppIII cycles depends on that between ${ }^{3} \mathrm{He}+{ }^{3} \mathrm{He}$, which is quadratic in $X_{3}$, and ${ }^{3} \mathrm{He}+$ ${ }^{4} \mathrm{He}$, which is linear. The downward flow with the velocity shown in Fig. 7 sweeps ${ }^{3}$ He-rich material deep into the core (where $X_{3}^{e q}$ is quite small and $\tau_{3}^{e q}$ quite short), leading to its ignition. The resulting out-of-equilibrium ${ }^{3} \mathrm{He}$ burning enhances the ppI cycle relative to ppII + ppIII in proportion to $X_{3} / X_{3}^{e q}$ ratio achieved at ignition. Second, the ${ }^{3} \mathrm{He}+{ }^{4} \mathrm{He}$ reactions that do occur take place at small $\mathrm{r}$ and high $\mathrm{T}$, where the ppII/ppIII branching ratio $\sim T^{-10}$ favors ppIII over ppII. Thus the net effect of the circulation is to strongly reduce $\phi\left({ }^{7} \mathrm{Be}\right)$ and somewhat reduce $\phi\left({ }^{8} \mathrm{~B}\right)$. If such mixing were to occur in the sun, the core temperature would be reduced to about $0.96 T_{c}^{\mathrm{SSM}}$ because ppI burning is more efficient.

The naive $T_{c}$ argument invoked in Section 2 to argue against nonstandard models is therefore not universally valid. But, in the context of steady-state models with standard microphysics, existing neutrino flux results seem to leave only one nonstandard model possibility open: core mixing on time scales of ${ }^{3} \mathrm{He}$ equilibration.

What is surprising about this possibility - given that no solar physics went into its deduction - is that it has some physical plausibility. The possibility of large-scale collective flow induced by the SSM ${ }^{3} \mathrm{He}$ gradient is a long-standing concern: even recent explorations of the known SSM "solar spoon" overstability conclude that mixing of some amplitude could result [18]. The continuous mixing envisioned in Fig. 7 is attractive in this regard, because there is no composition gradient working against the flow, as the core is kept homogeneous in $\mathrm{H}$ and ${ }^{4} \mathrm{He}$ by the mixing. Another interesting feature is the circulation time of a few $\cdot 10^{7}$ years. This reminds one of a concern, originally expressed by Roxburgh [19], that the sun's early convective core, arising from out-of-equilibrium burning of the CNO cycle over the first $10^{8}$ years of the main sequence, could then persist because of the growth of the ${ }^{3} \mathrm{He}$ gradient.

As the arguments Andrew Cumming and I made have been misconstrued by some, I would like to "summarize my points" clearly:

- It is obvious that the MSW mechanism is elegant and has profound implications. It is the solution to the solar neutrino problem I favor.

- However existing generic arguments that no nonstandard model modification can lead to the flux pattern of Eq. (1) appear to be overstated.

- In the case of steady state models with standard microphysics, existing neutrino flux measurements appear to have narrowed the possibilities to a single class, those with core mixing on timescales of ${ }^{3} \mathrm{He}$ equilibration. This is remarkable.

- Somewhat surprisingly, this class may also be the one with the most physical plausibility. The core mixing possibilities associated with the solar spoon overstability and a persistant convective core have been discussed for many years.

- The question of whether such core mixing would arise in a 3D (or 2D) solar model is, at this point, purely speculative. Conversely, the absence of mixing in the SSM is not a physics result, but a reflection of its 1D character, i.e., of assumptions made at the outset.

- If a viable model could be constructed, the differences with the SSM would have consequences for a range of astrophysical issues: galactic ${ }^{3} \mathrm{He}$ evolution, the evolution of solar-like stars along the color-magnitude diagram, and helioseismology. The last is likely to be a very difficult test for the proposed mixing scheme to satisfy, as the molecular weight 
profile in the solar core would be quite different from the standard solar model. However I have yet to see a published argument on this point that I would regard as definitive.

- The challenge to theory to construct a 3D hydrodynamic model of the sun is considerable. Therefore it is likely that SNO and Superkamiokande will prove a solution to the solar neutrino puzzle before theory progresses on this issue.

- At one point the "negative $\phi\left({ }^{7} \mathrm{Be}\right)$ " issue seemed to hint that the solution had to be particle physics. The most recent Superkamiokande result (306 days), $\phi\left({ }^{8} \mathrm{~B}\right)=2.44$ $\pm 0.066_{-0.09}^{+0.05} \cdot 10^{6} / \mathrm{cm}^{2} \mathrm{~s}$, may weaken this claim. The corresponding ${ }^{37} \mathrm{Cl}$ result of $2.55 \pm$ $0.25 \mathrm{SNU}$ yields, for $\sigma\left({ }^{8} \mathrm{~B}\right)=(1.11 \pm 0.05) \cdot 10^{-42} \mathrm{~cm}^{2}$, the limit $\phi\left({ }^{8} \mathrm{~B}\right) \lesssim(2.30 \pm 0.22 \pm$ $0.05) \cdot 10^{6} / \mathrm{cm}^{2} \mathrm{~s}$. Thus this accommodates all of the Superkamiokande range at $1 \sigma$ without the need for a negative $\phi\left({ }^{7} \mathrm{Be}\right)$. (Of course, one must make room for a small pep/CNO contribution to the ${ }^{37} \mathrm{Cl}$ experiment; but the somewhat lower Superkamiokande result helps reduce the difficulty of achieving this). This emphasizes how crucial SNO and low-energy Superkamiokande data will be to proving neutrino oscillations.

- Again, the physics possibility sketched here is not offered as a solution to the solar neutrino puzzle, but as an argument that a nonstandard solar model solution remains an open possibility. It is not my favorite solution, nor is it one I'm prepared to completely rule out at this time.

\section{BIG-BANG NUCLEOSYNTHESIS VS. THE R-PROCESS}

Despite the cautions expressed in the previous section, I would like to further explore the consequences of a neutrino physics resolution of the solar neutrino puzzle. As discussed in Section 3, a $\nu_{e} \rightarrow \nu_{\mu}$ explanation of the missing solar neutrinos is nicely compatible with a cosmologically interesting $m_{\nu_{\tau}}$. However, there is growing evidence that the cosmological role of the $\nu_{\tau}$ is limited by constraints from r-process nucleosynthesis. This is the theme of this second lecture.

Given the cosmological bent of the audience, I would like to begin by drawing parallels between big bang nucleosynthesis - a cornerstone of modern cosmology - and nucleosynthesis in a supernovae. In the big-bang one encounters:

- An expanding, radiation-dominated, proton rich gas. Below about $T \sim 1 \mathrm{MeV}$ the weak interactions have frozen out, prior to nucleosynthesis.

- A nuclear freezeout occurs at $T \sim 100 \mathrm{keV} \sim 10^{9} \mathrm{~K}$, when the $\mathrm{n}+\mathrm{p} \rightarrow \mathrm{d}+\gamma$ bottleneck is broken. The resulting ${ }^{4} \mathrm{He} / \mathrm{H}$ ratio depends on the $\mathrm{n} / \mathrm{p}$ ratio at freezeout $(\sim$ $1 / 7)$.

- The nuclear gas is relatively dilute, so three-body reactions are rare. The absence of stable nuclei at $\mathrm{A}=5$ and 8 terminates the reaction chains, as $\alpha+\alpha$ and $\alpha+\mathrm{p}$ then cannot proceed.

Li.

- Today a fossil record of the big bang exists in the abundances of $\mathrm{H},{ }^{3} \mathrm{He} / \mathrm{d},{ }^{4} \mathrm{He}$, and

- The clarity with which this primordial record can be read depends on our ability to correct for the effects of subsequent galactic chemical evolution. Figure 8, showing the complicating effects of both stellar production and destruction of $\mathrm{Li}$, is one illustration of the challenges. 
- Our resulting understanding of big bang nucleosynthesis has yielded fundamental constraints on cosmological and particle physics parameters, determining the baryon/photon ratio $\eta$ and constraining the number of light neutrino generations.

The less familiar (to this audience) conditions found near the mass cut of a Type II supernova are quite similar, yet the few differences have interesting consequences:

- This "hot bubble" region in a supernova is an expanding, radiation dominated, neutron rich gas. Neutrinos streaming through this region are thermally decoupled from the matter.

- Important nucleosynthesis occurs as this material expands off the protoneutron star, dropping in temperature from $\mathrm{T} \sim 300 \mathrm{keV}$ to $\mathrm{T} \sim 100 \mathrm{keV}$, where reactions freeze out. The $\mathrm{n} / \mathrm{p}$ ratio is crucial to this synthesis.

- The nucleon gas is sufficiently dense that reactions such as $3 \alpha \rightarrow{ }^{12} \mathrm{C}$ can bridge the mass gap at $\mathrm{A}=8$. The $\alpha$ process is thought to proceed up to medium mass nuclei, producing a gas dominated by ${ }^{4} \mathrm{He}$, a few heavy seed nuclei, and excess neutrons.

- The resulting rapid capture of neutrons on the seeds produces the r-process nuclei. The current abundances of these nuclei constitute a fossil record of past galactic supernova nucleosynthesis.

- An understanding of this synthesis can yield important constraints on particle physics and astrophysics parameters, such as the mass and mixing of the $\nu_{\tau}$ and the supernova rate averaged over the galaxy's lifetime.

The recent development is the convergence of several arguments which place the site of the r-process deep within Type II supernova. I will first describe the r-process, then review these arguments.

\section{THE R-PROCESS AND ITS ASTROPHYSICAL SITE}

Consider a stellar environment where a neutron gas is present together with nuclei. Within the nucleus the surface of the neutron/proton Fermi seas are $\sim 8 \mathrm{MeV}$ below the continuum, a value presumably much above stellar temperatures. We assume that the neutron capture rate $(n, \gamma)$ is slow compared to typical nuclear $\beta$ decay rates. This then allows the weak interaction to maintain the equilibrium of the proton/neutron Fermi seas as neutrons are captured. Thus the neutron-induced nucleosynthesis proceeds along a path in $(\mathrm{N}, \mathrm{Z})$ centered on the stable nuclei. This is called the s- or slow-process. The rate of nucleosynthesis, that is, the rate of change of $\mathrm{A}=\mathrm{N}+\mathrm{Z}$, is then controlled by the neutron capture rate.

The r- or rapid-process requires more exotic stellar conditions. The neutron capture rate is fast compared to $\beta$ decay and thus determines a new equilibrium condition: quantum levels above the usual neutron Fermi sea in the nucleus fill to within a distance $\propto$ T of the continuum, where $\mathrm{T}$ is temperature of the neutron gas, as $(\mathrm{n}, \gamma) \leftrightarrow(\gamma \mathrm{n})$ comes into balance. The nucleosynthesis rate is then proportional to the $\beta$ decay rate: any beta decay of a neutron to a proton opens up a hole in the neutron sea, which then is rapidly refilled. The nucleosynthesis path is along very exotic neutron-rich nuclei, determined by the $(\mathrm{n}, \gamma) \rightarrow(\gamma$, n) equilibrium. If a $\beta$ decay rate for a particular nucleus $(\mathrm{Z}, \mathrm{N})$ is slow, the mass flow is restricted at the point, increasing the abundance. Thus the abundance for $(\mathrm{Z}, \mathrm{N})$ is expected to be inversely proportional to the $\beta$ decay rate at that $\mathrm{Z}$ and $\mathrm{N}$. 
Nuclei exhibit gaps in their level structure at closed shells, e.g., $\mathrm{N} \sim 82$ and $\sim 126$. When such a gap is encountered, the mass flow, controlled by $\beta$ decay, is redirected along $\mathrm{N}=$ constant in the $(Z, N)$ plane, until the gain in $(Z, N)$ symmetry energy is sufficient to bridge the gap, bringing the next neutron level below the continuum. As $\beta$ decay rates near closed shells are low, the closed neutron shells produce large abundance peaks, as can be seen in Figs. 9 and 10.

Once the neutron exposure ends, the r-process products decay back to the valley of stability, $(\mathrm{Z}, \mathrm{N}) \rightarrow(\mathrm{Z}+\Delta, N-\Delta)$ by repeated $\beta$ decay. Spallation following $\beta$ decay can shift A to somewhat lower values.

About half of all nuclei above $\mathrm{A} \gtrsim 80$, including all the transuranics, are synthesized by the r-process. For example the s-process chain

$$
\begin{gathered}
(Z, N)_{e e}^{s}+n \rightarrow(Z, N+1)_{e o} \vec{\beta}(Z+1, N)_{o e}^{s}+n \rightarrow \\
(Z+1, N+1)_{o o} \underset{\beta}{\rightarrow}(Z+2, N)_{e e}^{s}+n \rightarrow(Z+2, N+1) \ldots
\end{gathered}
$$

flows through $\mathrm{A}=\mathrm{Z}+\mathrm{N}+1$ and $\mathrm{Z}+\mathrm{N}+2$, producing the stable odd-even and even-even isotopes $(Z+1, N)_{o e}^{s}$ and $(Z+2, N)_{e e}^{s}$, but bypasses the even-even nucleus $(\mathrm{Z}, \mathrm{N}+2)$ which frequently is stable. But this neutron-rich bypassed isotope would be produced by the rprocess. Thus some nuclei are uniquely due to the r-process, while others can be synthesized by both the s- and r-processes or only by the s-process.

It has been known for almost four decades that the r-process requires spectacularly explosive conditions

$$
\begin{aligned}
\rho_{n} & \sim 10^{20} / \mathrm{cm}^{3} \\
T & \sim(1-3) \cdot 10^{9} \mathrm{~K} \\
t & \sim 1 \mathrm{sec}
\end{aligned}
$$

where $\rho_{n}$ is the neutron density. Suggested primary sites - those requiring no pre-enrichment of s-process elements to serve as "seeds" for the neutron capture - include the neutronized atmospheres above the protoneutron stars in Type II supernova explosions, neutron-rich jets from supernovae or neutron star mergers, and inhomogeneous big bangs. Secondary sites those with pre-existing seeds - can support successful r-processes with somewhat lower $\rho_{n}$. Suggestions have included the He and $\mathrm{C}$ zones in Type II supernovae and the red giant He flash.

There is a growing body of evidence favoring a primary r-process in Type II supernova. The discovery of very metal poor halo stars, $[\mathrm{Fe} / \mathrm{H}] \sim-1.7$ and -3.12 , enriched in r-process material, argues for a primary process, already operating in the early history of the galaxy [21]. Studies of galactic chemical evolution [22] have found that the growth of r-process material is consistent with low-mass Type II supernovae being the r-process site. Finally, the suggestion made long ago that the r-process might be associated with the expansion and cooling of neutron-rich matter from the vicinity of the mass cut in supernovae [23] has been modeled much more convincingly. It has been shown in Ref. [24] that an expanding neutron-rich nucleon gas can undergo an $\alpha$-particle freezeout, on which effectively all of the protons are consumed, followed by an $\alpha$-process, in which seed nuclei near $\mathrm{A}=100$ are produced. The r-process then takes place through the capture of excess neutrons on these seeds. While this specific model has some shortcomings - overproduction of ${ }^{88} \mathrm{Sr},{ }^{89} \mathrm{Y}$, 
and ${ }^{90} \mathrm{Zr}$ and the need for very high entropies - it has demonstrated that a supernova "hot bubble" r-process can produce both a reasonable abundance distribution and an appropriate amount of r-process ejecta.

\section{SUPERNOVA NEUTRINOS AND THE R-PROCESS}

In the infall stage of a core collapse supernova, neutrinos are trapped once a density of $\sim 10^{12} \mathrm{~g} / \mathrm{cm}^{3}$ is reached, guaranteeing that the $3 \cdot 10^{53}$ ergs of released gravitational energy is locked within the core until after core bounce. Eventually $99 \%$ of this energy is released after core bounce in the form of neutrinos, which diffuse outward to the trapping radius (or neutrinosphere) on a time scale $\tau \sim 3$ sec. During this random walk the neutrinos remain in weak equilibrium through interactions of the type $\nu_{e} \bar{\nu}_{e} \leftrightarrow \nu_{\mu} \bar{\nu}_{\mu}$ which guarantees approximate equipartition of the energy per flavor. However the final decoupling of the neutrinos from the matter is flavor dependent due to the stronger $\nu_{e}+e^{-} \leftrightarrow \nu_{e}+e^{-}$cross section and the charge current reactions

$$
\begin{gathered}
\nu_{e}+n \leftrightarrow p+e^{-} \\
\bar{\nu}_{e}+p \leftrightarrow n+e^{+} .
\end{gathered}
$$

The net result is a characteristic hierarchy of temperatures

$$
\begin{gathered}
T_{\nu_{\mu}, \bar{\nu}_{\mu}, \nu_{\tau}, \bar{\nu}_{\tau}} \sim 8 \mathrm{MeV} \\
T_{\bar{\nu}_{e}} \sim 5 \mathrm{MeV} \\
T_{\nu_{e}} \sim 4 \mathrm{MeV}
\end{gathered}
$$

where the $\bar{\nu}_{e} / \nu_{e}$ difference is a result of the neutron richness of the matter, which enhances the reaction in (16a) and keeps the $\nu_{e}$ coupled until it reaches a somewhat larger radius and correspondingly lower $\mathrm{T}$.

Due to work by Woosley, Haxton, et al. [25] and by Domagatskii and collaborators [26], it has been appreciated that neutrinos, on passing through the mantle of the supernova, can be responsible for novel nucleosynthesis. A much discussed example is the production of ${ }^{19} \mathrm{~F}$ by $\left(\nu, \nu^{\prime}\right)$ spallation within the Ne zone. This is an effective method of synthesizing the ${ }^{19} \mathrm{~F}$ found in our galaxy because the ${ }^{19} \mathrm{~F} /{ }^{20} \mathrm{Ne}$ ratio is small, $\sim 0.0003$.

Now the neutrino process production of ${ }^{19} \mathrm{~F}$ occurs in the Ne shell, (2-3) $\cdot 10^{4} \mathrm{~km}$ from the protoneutron star. The "hot bubble" r-process, though occurring at $\sim 10$ sec after core bounce when the majority of neutrinos have already escaped, takes place at 600-1000 $\mathrm{km}$, where the neutrino flux is far more intense. Thus the question that occurred to my collaborators and me: Could there be a "neutrino finger print" on the r-process distribution that would prove it occurred in an intense neutrino flux? 
It is clear that neutrino effects during the r-process may be "erased" because A $\left(\nu, \nu^{\prime}\right) A^{*}$ reactions are masked by the much stronger effects of photoabsorption. Yet this leaves open the possibility of interesting postprocessing effects, occurring after the r-process has frozen out at $\mathrm{T} \sim 10^{9} \mathrm{~K}$. To assess these effects, we integrated backward in time, subtracting from the observed r-process distribution the effects of neutrino spallation following freezeout. This "inversion" produces, from the final r-process distribution found in nature, the "true" rprocess distribution at freezeout as a function of the assumed neutrino fluence after freezeout.

The results [27] are given in Figs. 10 and 11. The neutrinos have a general smoothing effect and dramatically change the distribution in two places, the "valleys" below the abundance peaks at $\mathrm{A} \sim 130$ and $\sim 195$. This is easily understood: the effects of neutrino postprocessing, where neutrino reactions transfer enough energy to typically knock 3-5 neutrons out of the neutron-rich target nucleus, are magnified in this region because the parent nuclei (in the abundance peaks) are very abundant. The analogy with ${ }^{19} \mathrm{~F}$ and ${ }^{20} \mathrm{Ne}$ is clear.

In particular, eight nuclei, lying in the windows $A=124-126$ and $183-187$, are inordinately sensitive to postprocessing. Initially we thought that, by demanding these nuclei not be overproduced by the postprocessing, we could only place an upper bound on the neutrino fluence following freezeout. These window isotopes are not produced at a significant level in most r-process calculations. We found, instead, that their abundances could be fit at $\sim 1 \sigma$ under the hypothesis of neutrino-induced nucleosynthesis, assuming a fluence slightly below our limiting value. The results are

$$
\begin{aligned}
& \mathcal{F}(A=124-126) \sim 0.031 \\
& \mathcal{F}(A=183-187) \sim 0.015
\end{aligned}
$$

where the fluences are per flavor and specified in units of $10^{51} \mathrm{ergs} /(100 \mathrm{~km})^{2}$. These values correspond closely to those of the "hot bubble" r-process [24]. The lower value of $\mathcal{F}$ for A $=183-187$ is natural, corresponding to later synthesis of the $\mathrm{A} \sim 195$ mass peak. Thus a "neutrino finger print" of the "hot bubble" r-process is found, providing another strong argument for a primary Type II supernova r-process.

\section{OSCILLATIONS OF TAUON NEUTRINOS}

In Section $3 \mathrm{I}$ argued that $\nu_{e} \rightarrow \nu_{\mu}$ oscillations are an attractive explanation of the solar neutrino problem, leaving a massive $\nu_{\tau}$ free to play an important role in cosmology as hot dark matter. But, extending Fig. 4 as shown in Fig. 11, this then leads to a second, $\nu_{e} \leftrightarrow \nu_{\tau}$ crossing at higher densities. For $m_{\nu_{\tau}}$ in the cosmologically interesting window of (1-50) eV, this crossing occurs outside the neutinosphere in a supernova, i.e., after the neutrinos have decoupled from the matter. The result is an interchange in the corresponding spectra, producing anomalously hot $\nu_{e} \mathrm{~s}$,

$$
T_{\nu_{e}}^{\mathrm{MSW}} \sim 8 \mathrm{MeV} \gg T_{\bar{\nu}_{e}}
$$

The $\bar{\nu}_{e}$ experiences no crossing.

Now the cross sections in Eqs. (16) vary as $\mathrm{T}^{2}$, while the luminosity is approximately equipartitioned in flavor. Consequently the rate for $\nu_{e}+n \rightarrow e^{-}+p$ in the hot bubble is 
approximately doubled if $\nu_{e} \rightarrow \nu_{\tau}$ oscillations occur, while the $\bar{\nu}_{e}+p \rightarrow e^{+}+n$ rate remains fixed. Thus the $\nu_{e} \rightarrow \nu_{\tau}$ crossing can drive the "hot bubble" proton rich, destroying any prospect for an r-process.

The necessary conditions for an adiabatic $\nu_{e} \leftrightarrow \nu_{\tau}$ crossing affecting the r-process have been given by Fuller and Qian [28]. The existence of a "hot bubble" r-process puts stringent limits on a cosmologically interesting $\nu_{\tau}$.

To make the discussion specific, let's suppose a $7 \mathrm{eV}$ neutrino is advocated by large-scale structure studies. What are the consequences?

- Such a $\mathrm{m}_{\nu_{\tau}}$ is acceptable for modest $\theta_{e \tau} \gtrsim 10^{-2}$ only if the above r-process argument is wrong. (That is, the r-process actually occurs elsewhere). I think this is unlikely. More important, this can be checked in terrestrial detectors by measuring $\mathrm{T}_{\nu_{e}}$ and $\mathrm{T}_{\bar{\nu}_{e}}$ for the neutrinos emitted by the next galactic supernova, an event we should not miss!

- The $\mathrm{m}_{\nu_{\tau}}$ is $\sim 7 \mathrm{eV}$ and the r-process argument is evaded by having a small mixing angle, $\theta_{e \tau} \lesssim 0.003$. This would mean that a $\nu_{e} \leftrightarrow \nu_{\tau}$ oscillation fails to occur in a supernova because the small mixing angle leads to a nonadiabatic crossing.

Now this is not implausible since such a $\theta_{e \tau}$ is consistent with possible ranges for thirdgeneration CKM angles

$$
\begin{aligned}
& 0.002<V_{u b}<0.007 \\
& 0.003<V_{t d}<0.018
\end{aligned}
$$

and thus fits with some (probably poorly justified) prejudices. But this has the ugly consequence that terrestrial $\nu_{e} \rightarrow \nu_{\tau}$ disappearance experiments $\left(\sin ^{2} 2 \theta_{e \tau} \lesssim 4 \cdot 10^{-5}\right)$ are then very difficult. The best hope to experimentally confirm a hypothesized hot dark matter $\nu_{\tau}$ would then be a more favorable $\theta_{\mu \tau}$, leading to a measurable $\nu_{\mu} \rightarrow \nu_{\tau}$ disappearance.

Of course, it could turn out the $\theta_{\mu \tau}$ is also quite small, frustrating oscillation searches in this channel, too. It then might prove difficult to demonstrate the existence of a cosmological interesting $\nu_{\tau}$ in the laboratory, a prospect large scale structure theorists might find annoying.

- Finally there is one other way out: the mixing angle could be large and the r-process argument correct, yet r-process consequences could be evaded by making $m_{\nu_{\tau}} \lesssim 3 \mathrm{eV}$. The necessary $7 \mathrm{eV}$ of dark matter could be achieved with $m_{\tau_{\nu}} \sim m_{\nu_{\mu}} \sim m_{\nu_{e}}$. This scenario has been recently invoked in order to make sense out of a variety of astrophysical and terrestrial hints of massive neutrinos [29].

The good news is that SNO and Superkamiokande should help us take the first step, deciding whether massive neutrinos are responsible for the solar neutrino problem. This will be a great help in reading the "tea leaves" of a cosmologically interesting $\nu_{\tau}$.

I thank the Ettore Majorana International Centre for hosting this school and Prof. Norma Sanchez for her invitation and able leadership of the school. The research reported here was supported by the US Department of Energy. 


\section{REFERENCES}

[1] Bahcall, J.N. and Pinsonneault, M.H. (1995) Rev. Mod. Phys., 67, 781.

[2] Turck-Chieze, S. and Lopes, I. (1993) Ap. J., 408, 347.

[3] Davis, R. Jr., Harmer, D.S. and Hoffman, K.C. (1968) Phys. Rev. Lett., 20, 1205; Lande, K. in Neutrino '94, A. Dar, G. Eilam and M. Groneau (eds.), Amsterdam, North Holland, 1994.

[4] Hirata, K.S. et al. (1988) Phys. Rev., D38, 448 and (1991) D44, 2241.

[5] Suzuki, Y., talk presented at this school.

[6] Abdurashitov, J.N. et al. (1994) Phys. Lett., B328, 234; (1995) Nucl. Phys. B, 38, 60; (1996) Phys. Rev. Lett., 77, 4708.

[7] Anselmann, P. et al. (1992) The GALLEX Collaboration, Phys. Lett. B, 285, 376; (1994) 327, 377; (1995) 342440.

[8] Ewan, G.T. et al. (1987) Queen's University report SNO-87-12, and (1995) Proc. IV International Symposium on Weak and Electromagnetic Interactions in Nuclei, H. Ejiri, T. Kishimoto and T. Sato (eds.), World Scientific, Singapore, pp. 647-53.

[9] Haxton, W.C. (1995) Ann. Rev. Astron. Astrophys., 33, 459.

[10] Hata, N., Bludman, S., and Langacker, P. (1994) Phys. Rev. D, 49, 3622; Hata, N. and Langacker, P. (1994) Phys. Rev. D, 48, 2937.

[11] Castellani, V., Degl'Innocenti S., Fiorentini, G., Lissia, M. and Ricci, B. (1994) Phys. Rev. D, 50, 4749.

[12] Mikheyev, S.P. and Smirnov, A. Yu. (1986) Nuovo Cimento, 9C, 17; (1989) Prog. in Part. and Nucl. Phys., 23, 41; Wolfenstein, L. (1978) Phys. Rev., D17 2369 and (1979) 20, 2634.

[13] Bethe, H. (1986) Phys. Rev. Lett., 56, 1305.

[14] Haxton, W.C. (1986) Phys. Rev. Lett., 57, 1271.

[15] Parke, S.J. (1986) Phys. Rev. Lett., 57, 1275.

[16] See, for example, Babu, K.S. and Mohopatra, R.N. (1993) Phys. Rev. Lett., 70, 2845, Bludman, S.A., Kennedy, D.C. and Langacker, P.G. (1992) Phys. Rev. D, 45, 1810; Dimopoulos, S., Hall, L.S. and Raby, S. (1993) Phys. Rev. D, 47, R3697.

[17] Cumming, A. and Haxton, W.C. (1996) Phys. Rev. Lett., 77, 4286.

[18] Dilke, F.W.W. and Gough, D.O. (1972) Nature, 240, 262; Ghosal, S. and Spiegel, E.A. (1991) Geophys. Astrophys. Fluid Dynamics, 61, 161.

[19] Roxburgh, I.W. (1985) Solar Physics, 100, 21.

[20] Timmes, F.X., Woosley, S.E. and Weaver, T.A. (1995) Ap. J. Suppl., 98, 617.

[21] Cowan, J.J., Burris, D.L., Sneden, C., McWilliam, A. and Preston, G.W. (1995) Astrophys. J., 439, L51; Cowan, J.J., Sneden, C., Truran, J.W. and Burris, D.L. (1996) Astrophys. J., 460, L115.

[22] See, for example, Matthews, G.J. and Cowan, J.J. (1990) Nature (London), 345, 491.

[23] Early work is reviewed in Hillebrandt, W. (1978) Space Sci. Rev., 21, 639.

[24] Woosley, S.E., Wilson, J.R., Mathews, G.J., Hoffman, R.D. and Meyer, B.S. (1994) Astrophys. J., 433, 229 and references therein.

[25] Woosley, S.E., Hartmann, D.H., Hoffman, R.D. and Haxton, W.C. (1990) Astrophys. J., 356, 272.

[26] Domogatsky, G.V. and Nadyazhin, D.K. (1978) Sov. Astron., 22, 297; (1980) Astrophys. Space Sci., 70, 55; (1980) Sov. Astron. Letters, 6, 127. 
[27] Haxton, W.C., Langanke, K., Qian, Y.-Z. and Vogel, P. (1997) Phys. Rev. Lett., 78, 2694 and (1997) Phys. Rev. C, 55, 1532.

[28] Qian, Y.-Z. et al. (1993) Phys. Rev. Lett., 71, 1965; Fuller, G.M. (1993) Phys. Rep., 227, 149.

[29] Cardall, C.Y. and Fuller, G.M. (1996) Phys. Rev. D, 53, 4421; Acker, A. and Pakvasa, S. (1997) Phys. Lett. B, 397, 209. 


\section{FIGURES}

FIG. 1. The solar pp chain. Note that each of the three cycles, ppI, ppII, and ppIII, can be associated with a distinct neutrino.

FIG. 2. The flux densities (solid lines) of the principal $\beta$ decay sources of solar neutrinos of the standard solar model. The total fluxes are those of the SSM of Ref. [1]. The ${ }^{7}$ Be and pep electron capture neutrino fluxes (dashed lines) are given in units of $\mathrm{cm}^{-2} \mathrm{~s}^{-1}$.

FIG. 3. The fluxes allowed by the combined results of the Homestake, SAGE/GALLEX, and Kamiokande experiments compared to the uncertainties of the SSM (upper ellipse) and to various nonstandard model predictions. The solid line is the $\mathrm{T}_{c}$ power law (From Ref. [10]).

FIG. 4. Schematic illustration of the MSW level crossing. The dashed lines correspond to the electron-electron and muon-muon elements of the mass matrix. Their intersection defines the level crossing density $\rho_{c}$. The solid lines are the trajectories of the local heavy and light mass eigenstates. If an electron neutrino with a suitable vacuum mass is produced deep in the solar core and propagates adiabatically, it will follow the heavy mass trajectory, emerging from the sun as a $\nu_{\mu}$.

FIG. 5. The MSW solutions allowed at 95\% confidence level, given the standard model fluxes of Ref. [1]. From Ref. [10].

FIG. 6. The dashed line represents the SSM ${ }^{3} \mathrm{He}$ profile after $\sim 4.6$ Gy of burning. The solid line indicates where ${ }^{3} \mathrm{He}$ would be burned given the convection pattern of Fig. 7.

FIG. 7. The convection pattern required to suppress both $\phi\left({ }^{8} \mathrm{~B}\right)$ and $\phi\left({ }^{7} \mathrm{Be}\right) / \phi\left({ }^{7} \mathrm{~B}\right)$. The downward flow is in plumes, rapid and localized, requiring $\sim$ few $\cdot 10^{6}$ years. This leads to

out-of-equilibrium burning of ${ }^{3} \mathrm{He}$ at small $\mathrm{r}$. The slow, broad upward flow allows the cycle to replenish the ${ }^{3}$ He. Typical upward times are $\sim$ few $10^{7}$ years.

FIG. 8. Li abundance in solar-like stars as a function of metallicity $[\mathrm{Fe} / \mathrm{H}]$, from Timmes et al. [20]. The region between the dashed curves represent the range of neutrino process contributions.

FIG. 9. The solid curve is the r-process production that, when combined with neutrino postprocessing, would produce the observed abundances (dashed line). It is assumed that the production in the window $\mathrm{A}=124-126$ is entirely due to postprocessing.

FIG. 10. As in Fig. 9, only for the A $~ 195$ mass peak. Again, the production in the window 183-187 is assumed to be due only to neutrino postprocessing. 
FIG. 11. The three-flavor level crossing diagram, analogous to Fig. 4, showing that a second crossing may occur at higher densities. 